\title{
Impact of the BDNF Val66Met Polymorphism on Regional Brain Gray Matter Volumes: Relevance to the Stress Response
}

\author{
Sung Nyun Kim ${ }^{1,3}$, Do-Hyung Kang ${ }^{1,4}$, Je-Yeon Yun ${ }^{1,4}$, Tae Young Lee, \\ Wi Hoon Jung ${ }^{3}$, Joon Hwan Jang ${ }^{1,4}$ and Jun Soo Kwon ${ }^{1,2,3,4} \bowtie$ \\ 1'Department of Psychiatry, Seoul National University College of Medicine, Seoul, Republic of Korea \\ ${ }^{2}$ Department of Brain and Cognitive Sciences - World Class University Program, Seoul National University College of Natural Sciences, Seoul, \\ Republic of Korea \\ ${ }^{3}$ Institute of Human Behavioral Medicine, SNU-MRC, Seoul, Republic of Korea \\ ${ }^{4}$ Department of Neuropsychiatry, Seoul National University Hospital, Seoul, Republic of Korea
}

\begin{abstract}
Objective Genetic imaging is used to investigate the mechanism by which genetic variants influence brain structure. In a previous study, a structural change of the dorsolateral prefrontal cortex was associated with symptom modulation in post-traumatic stress disorder patients. This study examined the effect of a polymorphism in the gene encoding brain-derived neurotrophic factor (BDNF) on regional gray matter (GM) volumes and the correlations between the dorsolateral prefrontal GM volume and the stress level in healthy volunteers.

Methods Sixty-one volunteers underwent genotyping for the BDNF Val66Met single nucleotide polymorphism (SNP) and completed the Stress Response Inventory (SRI). Magnetic resonance images were also acquired, and the effect of each subject's BDNF genotype and SRI subscore on his or her dorsolateral prefrontal GM volume was evaluated.

Results The Val/Val homozygotes had significantly larger GM volumes in the prefrontal cortex and the precuneus, the uncus, and the superior temporal and occipital cortices than Met carriers. The Met homozygotes demonstrated a higher stress response in depression domain than $\mathrm{Val} / \mathrm{Val}$ and $\mathrm{Val} / \mathrm{Met}$ groups. A negative correlation between the middle frontal cortex GM volume and the SRI depression subscore was found.

Conclusion These findings indicate an interaction between genes and brain structure, and they suggest that differences in dorsolateral prefrontal GM volume related to the BDNF Val66Met SNP are associated with resilience to stressful life events, particularly in the dimension of emotion.

Psychiatry Investig 2013;10:173-179
\end{abstract}

Key Words Brain-derived neurotrophic factor, Val66Met, Genetic polymorphism, Magnetic resonance imaging, Stress, Dorsolateral prefrontal cortex.

\section{INTRODUCTION}

Brain-derived neurotrophic factor (BDNF), a neurotrophin, plays essential roles in neuronal proliferation, differentiation, and survival during development and functions in the molecular mechanisms underlying neural plasticity and connectivity. In addition, BDNF is associated with neuronal plas-

Received: January 30, 2013 Revised: February 13, 2013

Accepted: February 13, 2013 Available online: May 30, 2013

$\triangle$ Correspondence: Jun Soo Kwon, MD, PhD

Department of Psychiatry, Seoul National University College of Medicine, 101 Daehak-ro, Jeon-gu, Seoul 110-744, Republic of Korea

Tel: +82-2-2072-2450, Fax: +82-2-747-9063, E-mail: kwonjs@snu.ac.kr

(a) This is an Open Access article distributed under the terms of the Creative Commons Attribution Non-Commercial License (http://creativecommons.org/licenses/by$\mathrm{nc} / 3.0$ ) which permits unrestricted non-commercial use, distribution, and reproduction in any medium, provided the original work is properly cited. ticity in response to stressful life events. Acute and chronic stresses increase BDNF expression in the basolateral amygdala. ${ }^{1}$ Moreover, BDNF itself stimulates hypothalamic hormone synthesis, ${ }^{2}$ which might be directly involved in the physiological response to stress. Additionally, BDNF-related stress resilience is conducted via the mesolimbic pathways, which are associated with bidirectional projections between the ventral tegmental area versus the nucleus accumbens and the prefrontal cortex (PFC). ${ }^{3}$ The PFC is a region of abundant expression among the many areas of the central nervous system in which BDNF is expressed.

An important polymorphism has been identified in codon 66 of the gene encoding BDNF, which is located on chromosome 11p14.1. This single nucleotide polymorphism (SNP) causes the substitution of methionine (Met) for valine (Val) 
(Val66Met). The Met allele has been linked with functional impairment of the hippocampus, including that of working memory performance. ${ }^{4}$ Additionally, the presence of the Met allele is associated with a decreased gray matter (GM) volume throughout the PFC, including the anterior cingulate cortex (ACC), ${ }^{5,6}$ the dorsolateral PFC (DLPFC), ${ }^{7}$ and the medial temporal lobes, which include the hippocampus, parahippocampal gyrus, and amygdala. ${ }^{8}$

Several recent studies have suggested that gene-stress interactions are mediated by regional brain volume differences according to the SNP allelic composition; the BDNF Val66Met SNP and DLPFC may be relevant candidates. In previous studies of subjects with post-traumatic stress disorder (PTSD) who had experienced traumatic stress, the structure and function of the DLPFC were reported to be altered; these changes were hypothesized to play an important role in modulating the fear and anxiety circuits. ${ }^{9}$ A recent neuroimaging study showed that greater DLPFC thickness is associated with greater PTSD symptom improvement and that the BDNF Val66Met gene polymorphism is associated with DLPFC thickness in PTSD patients. ${ }^{10}$ The authors concluded that the DLPFC thickness increased via the experience-dependent synaptic plasticity mediated by BDNF genetic variation in PTSD patients. However, the roles BDNF and the DLPFC play in mediating stress in healthy subjects remain unclear.

The present study examined regional GM volume differences in relation to the BDNF Val66Met SNP in normal subjects. Additionally, regarding the hypothesized role of the DLPFC in corticosteroid-related stress reactivity, which is under BDNF-related physiologic control, we analyzed the correlation between the DLPFC GM volume and the level of stress in 61 healthy volunteers. We sought to elucidate the structural neural correlate of the stress response in relation to the BDNF Val66Met SNP in the normal population.

\section{METHODS}

\section{Participants}

A total of 61 healthy volunteers (35 males and 26 females) were recruited as subjects and were evaluated using the Structured Clinical Interview for DSM-IV non-patient version. None of the subjects had been diagnosed with psychosis, bipolar disorder, major depressive disorder, substance abuse or dependence, significant head injury, seizure disorder, or an IQ lower than 70. This study was approved by the Institutional Review Board at Seoul National University Hospital, and written informed consent was obtained from all participants after the procedures had been fully explained. This study was conducted in accordance with the Declaration of Helsinki.

All participants completed the Stress Response Inventory
$(\mathrm{SRI}),{ }^{11}$ a self-report questionnaire that evaluates stress responses. The SRI includes 22 questions designed to address the following dimensions: somatization, depression, and anger. Each question was scored on a 5-point Likert scale, from 0 ("not at all") to 4 ("absolutely"). The reliability of the SRI was confirmed by measuring Cronbach's alpha (somatization, 0.89 ; depression, 0.88 ; and anger, 0.87$).{ }^{12}$

\section{Genotyping of BDNF}

The subjects' DNA was genotyped for the BDNF Val66Met SNP. Whole blood (12 mL) was drawn from each participant by venipuncture into EDTA-containing tubes. After measuring the blood volume, a $1-\mathrm{mL}$ aliquot was stored at $-20^{\circ} \mathrm{C}$ until genomic DNA extraction. Genomic DNA was isolated from $200 \mu \mathrm{L}$ of whole blood using a QIAamp DNA Blood Mini Kit (Qiagen, Hilden, Germany). The BDNF polymorphism was detected by TaqMan allelic discrimination assays using an ABI Prism 7500 Sequence Detection System (Applied Biosystems, Foster City, CA, USA), by which the BDNF Val66Met (rs6265, C_11592758_10) SNP was investigated. The reaction mixture contained $5 \mu \mathrm{L}$ of $2 \times$ TaqMan genotyping master mix, $0.5 \mu \mathrm{L}$ of $20 \times$ drug metabolism genotyping assay mix, $3.5 \mu \mathrm{L}$ of DNase-free water, and $1 \mu \mathrm{L}$ of genomic DNA in a total volume of $10 \mu \mathrm{L}$. The products were analyzed using ABI Prism 7500 Sequence Detection System software (version 2.0.1).

\section{Magnetic resonance image acquisition and preprocessing}

Magnetic resonance images were acquired on a 1.5 T Magnetom Avanto scanner (Siemens, Erlangen, Germany) using a T1-weighted magnetization-prepared rapid acquisition gradient echo sequence with the following imaging parameters: acquisition matrix $=256 \times 256$, voxel size $=0.45 \times 0.45 \times 0.90 \mathrm{~mm}^{3}$, axial slices $=176$, field of view $=23 \times 23 \mathrm{~cm}^{2}$, repetition time $=$ $1,160 \mathrm{~ms}$, echo time $=4.76 \mathrm{~ms}$, and no slice gap.

The raw DICOM magnetic resonance imaging (MRI) data were converted to the NIFTI format using SPM8 software (http://www.fil.ion.ucl.ac.uk/spm/software/spm8/) running on MATLAB R2010B (The MathWorks Inc., Natick, MA, USA). Brain volume normalizing, bias correcting, and segmentation into GM, white matter (WM), and cerebrospinal fluid (CSF) were performed with the VBM8 toolbox (http:// $\mathrm{dbm}$.neuro.uni-jena.de/vbm/), which is incorporated in SPM8. This method uses an optimized voxel-based morphometry (VBM) protocol and a model based on Hidden Markov Random Fields, which was developed to increase the signal-to-noise ratio. The total volumes of GM, WM, and CSF were calculated by adding the resulting tissue probabilities. Total brain volume was defined as the sum of the GM and 
Table 1. Demographic data, genetic polymorphism profile of subjects and the SRI scores

\begin{tabular}{|c|c|c|c|c|c|c|}
\hline & $\begin{array}{c}\text { Total } \\
(\mathrm{N}=61)\end{array}$ & $\begin{array}{c}\text { BDNF Val/Val } \\
(\mathrm{N}=20)\end{array}$ & $\begin{array}{c}\text { BDNF Val/Met } \\
(\mathrm{N}=29)\end{array}$ & $\begin{array}{c}\text { BDNF Met/Met } \\
(\mathrm{N}=12)\end{array}$ & $\mathrm{F}$ & $\mathrm{p}$ \\
\hline Age & $25.79 \pm 4.01$ & $24.95 \pm 4.55$ & $26.52 \pm 3.75$ & $25.42 \pm 3.66$ & 0.967 & 0.386 \\
\hline Sex (male/female) & $35 / 26$ & $14 / 6$ & $16 / 13$ & $5 / 7$ & 2.530 & 0.282 \\
\hline IQ & $114.07 \pm 10.26$ & $116.79 \pm 10.72$ & $113.82 \pm 7.96$ & $110.33 \pm 13.53$ & 1.497 & 0.233 \\
\hline SRI total score & $16.84 \pm 12.54$ & $13.85 \pm 10.69$ & $16.97 \pm 13.00$ & $21.50 \pm 13.77$ & 1.418 & 0.250 \\
\hline SRI somatization & $6.66 \pm 5.16$ & $5.70 \pm 4.78$ & $7.31 \pm 5.50$ & $6.67 \pm 5.09$ & 0.568 & 0.570 \\
\hline SRI depression & $6.49 \pm 6.17$ & $4.55 \pm 5.20$ & $6.00 \pm 5.56$ & $10.91 \pm 7.30$ & 4.682 & $0.013^{*}$ \\
\hline SRI anger & $2.13 \pm 1.94$ & $2.11 \pm 2.02$ & $2.00 \pm 1.79$ & $2.50 \pm 2.24$ & 0.280 & 0.757 \\
\hline
\end{tabular}

Means \pm SD. ${ }^{*} \mathrm{p}<0.05 / 3=0.017$. BDNF: brain-derived neurotrophic factor, Val: valine, Met: methionine, SRI: stress response inventory

WM volumes. Afterwards, the volume images were smoothed using an isotropic Gaussian kernel (full width at half maximum=8 mm).

\section{Statistical analysis}

Chi-square tests were performed to verify Hardy-Weinberg equilibrium and to compare the allelic and genotypic frequencies.

Analysis of variance (ANOVA) was used to compare demographic data and three SRI subscores (somatization, depression, and anger) among the Val/Val, Val/Met, and Met/ Met groups, and a post-hoc analysis was conducted for pairwise comparisons between each genotype group.

Voxelwise comparisons was applied to the GM images using genotype (Val/Val homozygotes vs Met+ carriers) as the factor and age and sex as covariates. All statistical tests for GM imaging were executed with a threshold of uncorrected $\mathrm{p}<0.005$ and $\mathrm{k}>50$. For the region of interest (ROI) analysis, statistically significant clusters showing the greater brain volume of the Val/Val group compared to the Met+ group were extracted using the MarsBaR toolbox (version 0.42).

Correlations between the SRI subscores and ROI cluster of the dorsolateral prefrontal cortex were also investigated. The significance threshold was set at $\mathrm{p}<0.05$, and a Bonferroni correction $(\mathrm{p}<0.017)$ was applied to the SRI subscores ANOVA and correlation analysis. Tukey's test was used for a posthoc pairwise analysis. All analyses were conducted using PASW version 18.0 (Statistical Package for the Social Sciences, SPSS Inc., Chicago, IL, USA) and SPM8 (Statistical Parametric Mapping, Wellcome Department, University College of London, UK, http://www.fil.ion.ucl.ac.uk/spm).

\section{RESULTS}

\section{Demographic and genetic characteristics}

Our study included 61 subjects in total and grouped them according to BDNF genotype into $20 \mathrm{Val}$ allele homozygotes

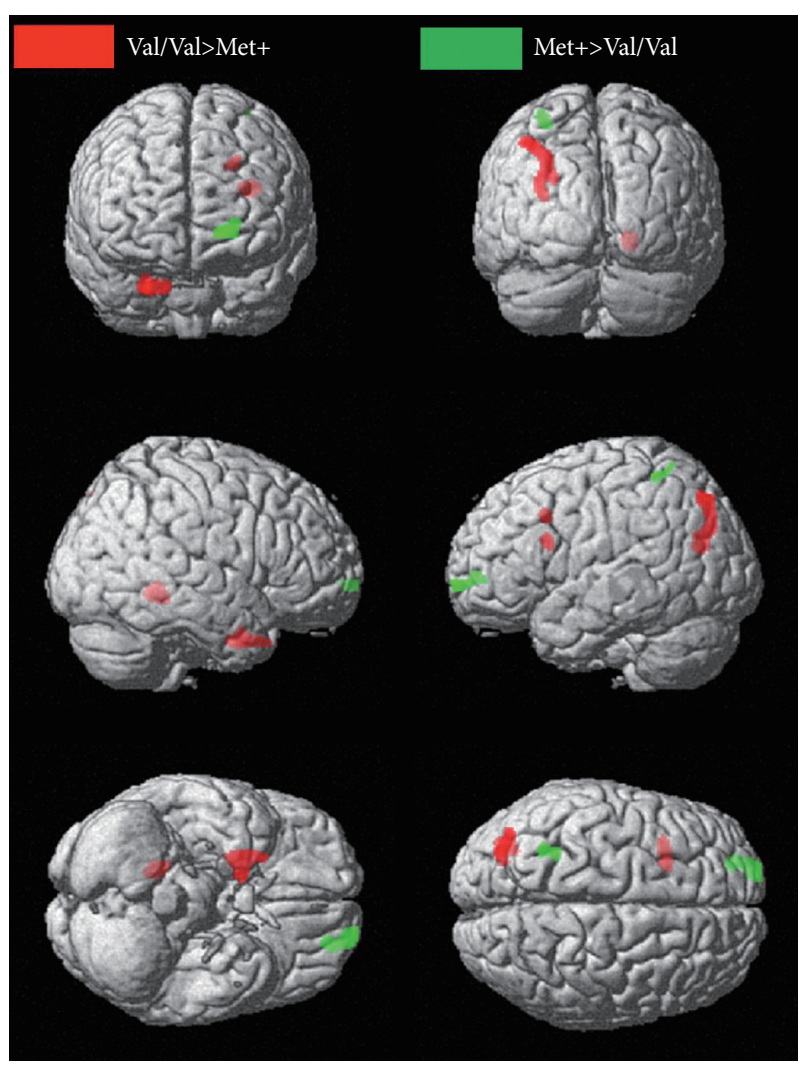

Figure 1. Significant differences in gray matter volume in relation to the BDNF SNP projected on a standard brain template (Significance thresholds: uncorrected $p<0.005$ and $k>50$ ). BDNF: brainderived neurotrophic factor, Val: valine, Met: methionine, SNP: single nucleotide polymorphism.

(Val/Val), 29 heterozygotes (Val/Met), and 12 Met allele homozygotes (Met/Met). The frequency of the BDNF genotypes was $32.8 \%$ for Val/Val, $47.5 \%$ for Val/Met, and $19.7 \%$ for Met/ Met in our sample. There were $20 \mathrm{Val}$ homozygotes and 41 Met allele carriers. There were no statistically significant differences in age, sex, or IQ between the genotype groups (Table $1)$. No significant deviation from Hardy-Weinberg equilibrium was found $\left(\chi^{2}=0.06, \mathrm{p}>0.05\right)$. 


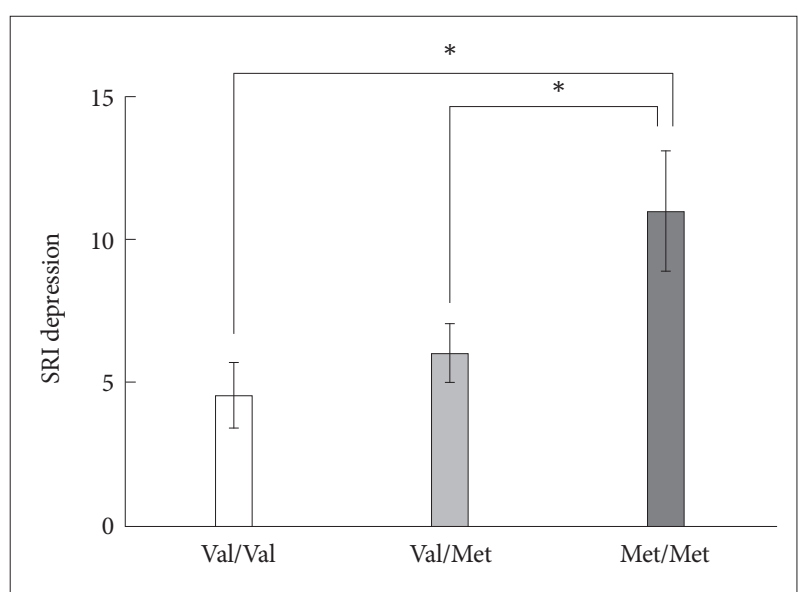

Figure 2. Significant differences in SRI depression scores according to BDNF SNP genotypes. ${ }^{*} p<0.05$, multiple comparison corrected. SRI: stress response inventory, BDNF: brain-derived neurotrophic factor, SNP: single nucleotide polymorphism, Val: valine, Met: methionine.

\section{Effect of BDNF genotype on regional brain GM volumes}

VBM revealed regional brain GM differences between the two groups (Val/Val and Met+)(Table 2). In the DLPFC region, left inferior frontal gyrus (BA 45) and middle frontal gyrus (BA 9) were smaller in Met+ group. Met+ carriers also had smaller GM volumes in the right culmen of the cerebellum, right uncus of the basal ganglia (BA 36 and 28), right superior temporal gyrus (BA 38), left superior occipital gyrus (BA 19), left precuneus (BA 19), and left superior parietal lobule (BA 7) than Val homozygotes. In contrast, the regional GM volumes of the left superior frontal gyrus (BA 10) and left superior parietal lobule (BA 7) were larger in the Met+ group (uncorrected $\mathrm{p}<0.005, \mathrm{k}>50$ ) (Figure 1 ).

\section{Effect of BDNF genotype on the stress response}

Groups of different BDNF genotypes showed a significant difference on the SRI depression subscore $(\mathrm{F}=4.682, \mathrm{p}=0.013)$ (Figure 2). Met/Met homozygotes had a higher SRI depression subscore than $\mathrm{Val} / \mathrm{Val}$ homozygotes $(\mathrm{p}=0.011$, Tukey corrected) and Val/Met heterozygotes ( $\mathrm{p}=0.044$, Tukey corrected) in the post-hoc analysis. Regarding the somatization and anger SRI scores, no statistically significant differences were found according to the genotypes.

\section{Correlations between the SRI subscores and ROIs}

We selected a significant cluster from the left inferior frontal and middle frontal cortex as ROIs to further investigate the correlations between the three SRI subscores (somatization, depression, and anger) and GM volume of the DLPFC. A statistically significant correlation was found between the SRI depression subscore and the left middle frontal GM volume for all 61 subjects [Pearson's correlation coefficient=

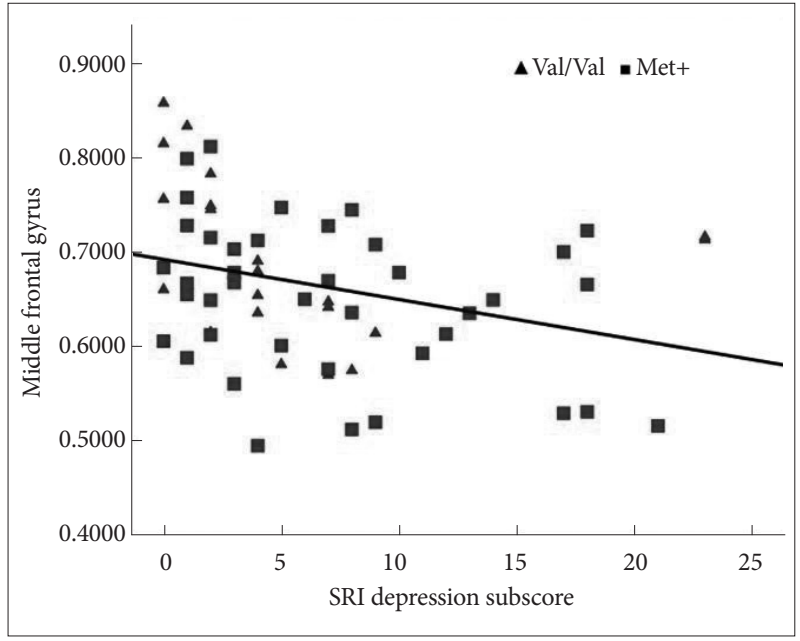

Figure 3. Scatterplot depicting the distributions of the SRI depression subscore and the brain GM volume of the left middle frontal gyrus in all subjects. Solid line: trend of the correlation between the two variables. SRI: stress response inventory, GM: gray matter, Val: valine, Met: methionine.

$-0.313, \mathrm{p}=0.014 ; \mathrm{p}<0.05 / 3$ (Bonferroni corrected)](Figure 3, Table 3). There were no significant correlations between the inferior frontal gyrus GM volume and the SRI subscores.

\section{DISCUSSION}

In this study, we aimed to elucidate the structural neural correlate of stress resilience in relation to the BDNF Val66Met polymorphism in normal subjects. We found that Met/Met homozygotes had a higher stress response in the depression domain (SRI depression subscore) compared to the $\mathrm{Val} / \mathrm{Val}$ and Val/Met groups. By investigating whole-brain GM volume differences according to the BDNF Val66Met SNP, we found that brain areas such as the middle frontal gyrus, the inferior frontal gyrus, precuneus, uncus, and superior temporal cortex had smaller GM volumes in the BDNF Met carriers compared to Val homozygotes. Additionally, we found a negative correlation between the GM volume of the middle frontal cortex (BA 9) and the stress response measured by the SRI depression subscore. To our knowledge, this is one of the first reports to demonstrate that the BDNF Val66Met SNP mediates resilience to stressful life events by influencing regional GM volume, particularly in the DLPFC region.

The middle and inferior frontal cortices exhibited greater GM volumes in the Val homozygotes compared to the Met allele carriers in this study. This result is in line with those of a previous VBM study targeting patients with bipolar disorder, which reported smaller GM volumes in the DLPFC and hippocampus in Val/Met bipolar patients compared with Val homozygote patients. ${ }^{13}$ In addition, the presence of the Met allele was reported to be associated with a more profound and 
Table 2. Regional differences in brain gray matter volume between BDNF Val homozygote group ( $N=20)$ and BDNF Met+group ( $N=41$ )*

\begin{tabular}{|c|c|c|c|c|c|c|c|c|c|}
\hline \multirow{2}{*}{ Brain area } & \multirow{2}{*}{ Laterality } & \multirow{2}{*}{ BA } & \multicolumn{3}{|c|}{ MNI coordinates } & \multirow{2}{*}{$\mathrm{T}$} & \multirow{2}{*}{$\mathrm{Z}$} & \multirow{2}{*}{ Cluster size } & \multirow{2}{*}{$\mathrm{p}$ value } \\
\hline & & & $\mathrm{x}$ & $\mathrm{y}$ & $\mathrm{z}$ & & & & \\
\hline \multicolumn{10}{|l|}{ Val/Val>Met+ } \\
\hline Middle frontal gyrus & $\mathrm{L}$ & 9 & -24 & 15 & 36 & 3.29 & 3.14 & 72 & 0.001 \\
\hline Triangular part of inferior frontal gyrus & $\mathrm{L}$ & 45 & -30 & 15 & 22 & 3.35 & 3.18 & 124 & 0.001 \\
\hline Superior occipital gyrus & $\mathrm{L}$ & 19 & -33 & -72 & 22 & 3.44 & 3.27 & 575 & 0.001 \\
\hline Precuneus & $\mathrm{L}$ & 19 & -29 & -78 & 39 & 3.34 & 3.18 & & 0.001 \\
\hline Superior parietal lobule & $\mathrm{L}$ & 7 & -41 & -75 & 49 & 3.23 & 3.08 & & 0.001 \\
\hline Culmen of cerebellum & $\mathrm{R}$ & - & 18 & -43 & -8 & 3.81 & 3.58 & 250 & $<0.001$ \\
\hline Uncus & $\mathrm{R}$ & 36 & 26 & 2 & -33 & 3.52 & 3.34 & 427 & $<0.001$ \\
\hline Uncus & $\mathrm{R}$ & 28 & 17 & 5 & -33 & 3.23 & 3.08 & & 0.001 \\
\hline Superior temporal gyrus & $\mathrm{R}$ & 38 & 26 & 15 & -33 & 3.05 & 2.93 & & 0.002 \\
\hline \multicolumn{10}{|l|}{$\mathrm{Met}+>\mathrm{Val} / \mathrm{Val}$} \\
\hline Superior parietal lobule & $\mathrm{L}$ & 7 & -29 & -46 & 58 & 3.29 & 3.13 & 88 & 0.001 \\
\hline Superior frontal gyrus (OFC) & $\mathrm{L}$ & 10 & -24 & 56 & 3 & 3.27 & 3.12 & 273 & 0.001 \\
\hline Superior frontal gyrus & $\mathrm{L}$ & 10 & -20 & 66 & -2 & 3.22 & 3.08 & & 0.001 \\
\hline
\end{tabular}

${ }^{*} \mathrm{p}<0.005$ and $\mathrm{k}>50$. BDNF: brain-derived neurotrophic factor, Val: valine, Met: methionine, BA: Brodmann area, MNI: Montreal Neurological Institute, OFC: orbitofrontal cortex

Table 3. Partial correlation analyses showing degrees of correlation between SRI and gray matter volume of left middle frontal gyrus in the whole subjects $(\mathrm{N}=61)$

\begin{tabular}{llccc}
\hline & & SRI somatization & SRI depression & SRI anxiety \\
\hline GM volume of Lt MFG & Pearson's correlation coefficient & -0.296 & -0.313 & -0.094 \\
& p value (two-tailed) & 0.020 & $0.014^{*}$ & 0.473 \\
\hline
\end{tabular}

${ }^{*} \mathrm{p}<0.05 / 3=0.017$. SRI: stress response inventory, GM: gray matter, MFG: middle frontal gyrus

widespread reduction in the bilateral DLPFC GM volume in a healthy aged population. ${ }^{14}$ The DLPFC has been identified as the neural correlate of psychological recovery from a severely stressful event because the degree of cortical thickness enrichment in the DLPFC area after trauma exposure could successfully predict the reduction in PTSD symptoms. ${ }^{10}$ In particular, we showed a correlation between a greater middle frontal gyrus (BA 9) GM volume and the lower SRI depression subscore. This result is in line with a previous functional MRI (fMRI) study of acutely traumatized individuals using a script-driven trauma imagery paradigm, in which BOLD activation of the middle frontal cortex (BA 47) was correlated with resilience to the stressful event. ${ }^{15}$ In another fMRI study using the emotional face paradigm, DLPFC hypo-activation was significantly correlated with the degree of cognitive vulnerability to stress, in-line with the diathesis-stress theory of depression. ${ }^{16}$

We found that several posterior brain regions were correlated with the BDNF SNP and could function as neural correlates of various stress reactions. In the 99m-HMPAO SPECT study, which compared patients with PTSD and trauma-exposed non-symptomatic controls, differences in HMPAO tracer distribution were found in the uncus. ${ }^{17}$ Likewise, recall of trauma-related autobiographic memory in PTSD patients was related to increased cerebral blood flow in the precuneus, ${ }^{18}$ which was negatively correlated with the WHO-10 score, a measure of current subjective well-being. In the temporal area, cortical thinning in the right superior temporal gyrus of subjects with PTSD was associated with an M50 auditory sensory gating deficit. ${ }^{19}$ The superior temporal and occipital (BA 19) cortices also showed increased activation in traumatic script-driven dissociative responses in PTSD patients who had been sexually abused..$^{20}$

The superior parietal lobule (SPL) is regarded as a component of the mirror neuron system and also as a neural correlate of analytical reasoning, including cognitive coping strategies for stressful life events. ${ }^{21,22}$ In this study, a group comparison according to the BDNF Val66Met SNP revealed diverse GM thicknesses along the rostro-caudal axis of the left SPL (BA 7). Specifically, the GM volume of the posterior portion of the SPL (7P) was thicker in the Val homozygote group, whereas that of the SPL anterior portion (7A) was thicker in the Met+ group. The left SPL area showed decreased activation during exposure to an emotional stress paradigm in a previ- 
ous fMRI study of somatoform pain disorder patients. ${ }^{23}$ This result needs further investigation.

This study had several limitations that should be considered. First, we did not consider the presence and intensity of previous stressful life events, despite the possible effect of BDNF expression. Likewise, physiologic responses to stressful events, including increased cortisol levels, could have a deleterious effect on regional brain volumes, including those of the hippocampus and prefrontal cortices..$^{24,25}$ Second, we had to merge the Val/Met and Met/Met groups as Met carriers in the GM analysis because of the small number of Met homozygotes $(n=12)$. Recently, an MRI study of Met/Met individuals argued that they had a wider GM difference than Val/Met heterozygotes compared to Val/Val homozygotes. ${ }^{26}$ Due to the small allele frequency of Met, further research is needed with a larger sample size.

In this VBM-based study of regional GM volume differences according to the BDNF Val66Met SNP, we found that the dorsolateral prefrontal region and multiple posterior brain regions in the cerebellum, visual association cortex, superior temporal cortex, uncus, and precuneus showed significantly decreased GM volumes in the Met+ carriers compared to Val homozygotes. Met/Met homozygotes demonstrated a higher stress response in the depression domain compared to the $\mathrm{Val} / \mathrm{Val}$ and $\mathrm{Val} / \mathrm{Met}$ groups. Moreover, a negative correlation was found between the GM volume of the DLPFC and the SRI depression subscore, suggesting that regional GM volume differences according to the BDNF Val66Met SNP are associated with resilience to stressful life events, especially in the dimension of mood regulation. A future study exploring the interaction between BDNF expression, stressful life events, regional brain volumes, and resilience to stressful events should help elucidate these complex gene-environment interactions.

\section{Acknowledgments}

This study was supported by the National Research Foundation of Korea (NRF) Grant funded by the Korean government (MEST) (no. 2011-0015639).

\section{REFERENCES}

1. Lakshminarasimhan $\mathrm{H}$, Chattarji S. Stress leads to contrasting effects on the levels of brain derived neurotrophic factor in the hippocampus and amygdala. PLoS one 2012;7:e30481.

2. Givalois L, Arancibia S, Alonso G, Tapia-Arancibia L. Expression of brain-derived neurotrophic factor and its receptors in the median eminence cells with sensitivity to stress. Endocrinology 2004;145:47374747.

3. Fanous S, Hammer RP Jr, Nikulina EM. Short- and long-term effects of intermittent social defeat stress on brain-derived neurotrophic factor expression in mesocorticolimbic brain regions. Neuroscience 2010; 167:598-607.

4. Richter-Schmidinger T, Alexopoulos P, Horn M, Maus S, Reichel M, Rhein $\mathrm{C}$, et al. Influence of brain-derived neurotrophic-factor and apoli- poprotein E genetic variants on hippocampal volume and memory performance in healthy young adults. J Neural Transm 2011;118:249257.

5. Gerritsen L, Tendolkar I, Franke B, Vasquez AA, Kooijman S, Buitelaar J, et al. BDNF Val66Met genotype modulates the effect of childhood adversity on subgenual anterior cingulate cortex volume in healthy subjects. Mol Psychiatry 2012;17:597-603.

6. Montag C, Weber B, Jentgens E, Elger C, Reuter M. An epistasis effect of functional variants on the BDNF and DRD2 genes modulates gray matter volume of the anterior cingulate cortex in healthy humans. Neuropsychologia 2010;48:1016-1021.

7. Pezawas L, Verchinski BA, Mattay VS, Callicott JH, Kolachana BS, Straub RE, et al. The brain-derived neurotrophic factor val66met polymorphism and variation in human cortical morphology. J Neurosci 2004;24:10099-10102.

8. Montag C, Weber B, Fliessbach K, Elger C, Reuter M. The BDNF Val66Met polymorphism impacts parahippocampal and amygdala volume in healthy humans: incremental support for a genetic risk factor for depression. Psychol Med 2009;39:1831-1839.

9. Hartley CA, Phelps EA. Changing fear: the neurocircuitry of emotion regulation. Neuropsychopharmacology 2010;35:136-146.

10. Lyoo IK, Kim JE, Yoon SJ, Hwang J, Bae S, Kim DJ. The neurobiological role of the dorsolateral prefrontal cortex in recovery from trauma. Longitudinal brain imaging study among survivors of the South Korean subway disaster. Arch Gen Psychiatry 2011;68:701-713.

11. Koh KB, Park JK, Kim CH, Cho S. Development of the stress response inventory and its application in clinical practice. Psychosom Med 2001; 63:668-678.

12. Choi SM, Kang TY, Woo JM. Development and validation of a modified form of the stress response inventory for workers. J Korean Neuropsychiatr Assoc 2006;45:541-553.

13. Matsuo K, Walss-Bass C, Nery FG, Nicoletti MA, Hatch JP, Frey BN, et al. Neuronal correlates of brain-derived neurotrophic factor Val66Met polymorphism and morphometric abnormalities in bipolar disorder. Neuropsychopharmacology 2009;34:1904-1913.

14. Nemoto K, Ohnishi T, Mori T, Moriguchi Y, Hashimoto R, Asada T, et al. The Val66Met polymorphism of the brain-derived neurotrophic factor gene affects age-related brain morphology. Neurosci Lett 2006;397: 25-29.

15. Daniels JK, Hegadoren KM, Coupland NJ, Rowe BH, Densmore M, Neufeld RW, et al. Neural correlates and predictive power of trait resilience in an acutely traumatized sample: a pilot investigation. J Clin Psychiatry 2012;73:327-332.

16. Zhong M, Wang X, Xiao J, Yi J, Zhu X, Liao J, et al. Amygdala hyperactivation and prefrontal hypoactivation in subjects with cognitive vulnerability to depression. Biol Psychol 2011;88:233-242.

17. Pagani M, Hogberg G, Salmaso D, Nardo D, Sundin O, Jonsson C, et al. Effects of EMDR psychotherapy on 99mTc-HMPAO distribution in occupation-related post-traumatic stress disorder. Nucl Med Commun 2007;28:757-765.

18. Nardo D, Hogberg G, Flumeri F, Jacobsson H, Larsson SA, Hallstrom T, et al. Self-rating scales assessing subjective well-being and distress correlate with rCBF in PTSD-sensitive regions. Psychol Med 2011;15:1-13.

19. Hunter M, Villarreal G, McHaffie GR, Jimenez B, Smith AK, Calais LA, et al. Lateralized abnormalities in auditory M50 sensory gating and cortical thickness of the superior temporal gyrus in post-traumatic stress disorder: preliminary results. Psychiatry Res 2011;191:138-144.

20. Lanius RA, Williamson PC, Boksman K, Densmore M, Gupta M, Neufeld RW, et al. Brain activation during script-driven imagery induced dissociative responses in PTSD: a functional magnetic resonance imaging investigation. Biol Psychiatry 2002;52:305-311.

21. Moriguchi Y, Ohnishi T, Decety J, Hirakata M, Maeda M, Matsuda H, et al. The human mirror neuron system in a population with deficient self-awareness: an fMRI study in alexithymia. Hum Brain Mapp 2009; 30:2063-2076. 
22. Tsujii T, Sakatani K, Masuda S, Akiyama T, Watanabe S. Evaluating the roles of the inferior frontal gyrus and superior parietal lobule in deductive reasoning: an rTMS study. Neuroimage 2011;58:640-646.

23. Stoeter P, Bauermann T, Nickel R, Corluka L, Gawehn J, Vucurevic G, et al. Cerebral activation in patients with somatoform pain disorder exposed to pain and stress: an fMRI study. Neuroimage 2007;36:418-430.

24. Carrion VG, Weems CF, Richert K, Hoffman BC, Reiss AL. Decreased prefrontal cortical volume associated with increased bedtime cortisol in traumatized youth. Biol Psychiatry 2010;68:491-493.

25. Mondelli V, Pariante CM, Navari S, Aas M, D’Albenzio A, Di Forti M, et al. Higher cortisol levels are associated with smaller left hippocampal volume in first-episode psychosis. Schizophr Res 2010;119:75-78.

26. Yang X, Liu P, Sun J, Wang G, Zeng F, Yuan K, et al. Impact of brain-derived neurotrophic factor Val66Met polymorphism on cortical thickness and voxel-based morphometry in healthy Chinese young adults. PLoS one 2012;7:e37777. 